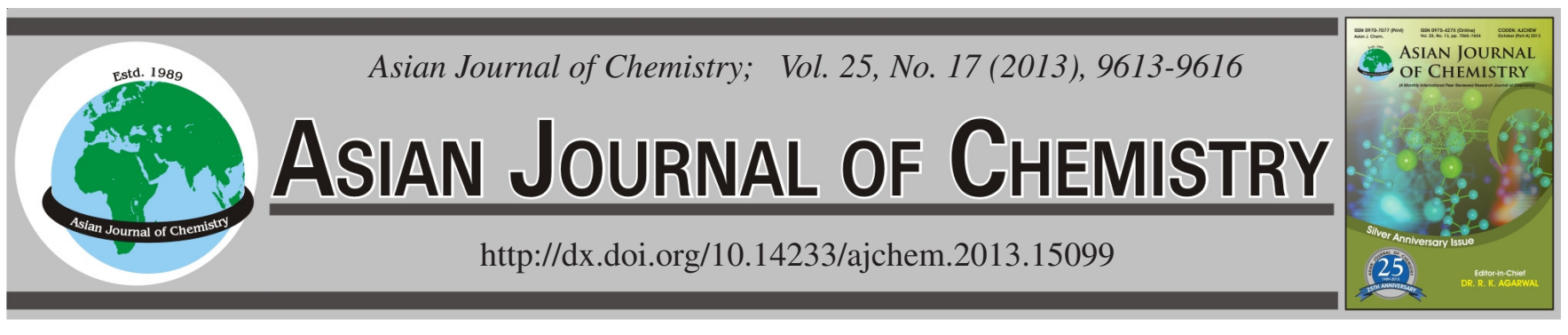

\title{
Synthesis and Characterization of Chitosan Human Like Collagen/ß-Sodium Glycerophosphate-Carbodiimide Hydrogel
}

\author{
Xian $\mathrm{Li}^{1}$, Dai-Di Fan ${ }^{1, *}$, Jian-Jun Deng ${ }^{1}$, Jun-Feng Hui ${ }^{1}, \mathrm{XiaO}_{\mathrm{i}}$-Xuan Ma ${ }^{1}$, Chen-Hui Zhu ${ }^{1}$ and Wen-Jiao Xue ${ }^{2}$
}

${ }^{1}$ Shaanxi Key Laboratory of Degradable Biomedical Materials, Department of Chemical Engineering, Northwest University, Taibai North Road 229, Xi'an 710069, Shaanxi Province, P.R. China

${ }^{2}$ Shaanxi Provincial Institute of Microbiology, No. 76 Xiying Road, Xi'an 710043, P.R. China

*Corresponding author: Fax: +86 29 88322585; Tel: +86 29 88305118; E-mail: fandaidi@ nwu.edu.cn

\begin{abstract}
Injectable in situ forming biodegradable chitosan-human like collagen (CS-HLC) based hydrogels has proved to be a potential candidate as an injectable biomaterial for tissue engineering. With crosslinking agent of carbodiimide, the properties of the novel chitosan-humanlike collagen/ $\beta$-sodium glycerophosphate-carbodiimide hydrogel were also examined. The gelation time, structure, equilibrium swelling and degradation in vitro and in vivo were dependent upon crosslinking and structure of composite hydrogels. The chitosan-human-like collagen/ $\beta$-sodium glycerophosphate-carbodiimide hydrogel showed a desirable gelling time, swelling ratio, a smooth surface, regular porous networks and biodegradable. Therefore, the chitosan-human-like collagen/ $\beta$-sodium glycerophosphate-carbodiimide hydrogels are excellent candidates for use in biomedical fields, such as in soft tissue defect filling and drug delivery.
\end{abstract}

Key Words: Biodegradable, Chitosan, Human-like Collagen, Carbodiimide, $\beta$-Sodium glycerophosphate.

\section{INTRODUCTION}

Various hydrogels and microspheres have been employed as injectable scaffolds for a variety of biomedical applications ${ }^{1-4}$. Injectable hydrogel is one of the most widely-used biomaterials for the treatment of soft tissue restoration. It is suitable for soft tissue defect filling, such as skin patches, wrinkles and tissue cavities caused by surgery. From a clinical perspective, the use of an injectable in situ forming hydrogel is more attractive than a preformed hydrogel as it can minimize patient discomfort, risk of infection, scar formation and cost of treatment ${ }^{5}$. Chitosan (CS), a partially deacetylated derivative from chitin composed of glucosamine and $\mathrm{N}$-acetylglucosamine, has been widely applied in drug delivery, gene therapy and tissue engineering because of its biocompatibility and biodegradability $^{6-8}$. However, chitosan has poor solubility in physiological solvents due to its strong intermolecular hydrogen bonding, thereby greatly limiting further biomedical applications, particularly as an injectable scaffold. Human-like collagen (HLC), a recombinant protein whose mRNA is derived from human, is highly expressed by recombinant Escherichia coli BL2 $1^{9,10}$. Because of its water solubility, poor rejection, workability, good biocompatibility, biodegradability and no risk of viral infection, HLC has been used in studies of artificial bone ${ }^{11,12}$, vascular scaffolds ${ }^{12}$ and chelated calcium ${ }^{13}$. The formation of a hydrogel, either by chemical cross-linking, heat or irradiation has been well described in the literature. Carbodiimide (EDC) is a water-soluble cross-linking agent, containing $\mathrm{N}=\mathrm{C}=\mathrm{N}$ functional group, show biocompatibility and long-term retention in vivo. This paper will be confined to chemical crosslinked hydrogels, which can be prepared based on chitosan/ human-like collagen (CS/HLC) based hydrogel.

The objective of the present was to prepare a non-toxic in situ forming biodegradable chitosan-human-like collagen/ $\beta$ sodium glycerophosphate-carbodiimide (CS-HLC/ $\beta$-GP-EDC) composite hydrogel and to study the effects of morphology, gelation time, equilibrium swelling and degradation in vivo and in vitro.

\section{EXPERIMENTAL}

The required HLC was expressed by $E$. coli with a cloned partial cDNA reversed from the mRNA coding for human collagen $^{14,15}$ (Chinese patent number: ZL01106757.8). The CS (molecular weight 550,000, deacetylation degree 90-92\%) was supplied by Qingdao Ocean Ltd. Co. (China). The $\beta$-sodium glycerophosphate ( $\beta$-GP), EDC and glacial acetic acid were also supplied by Sigma Co and all solvents and reagents were analytical grade.

Preparation of the CS-HLC/ $\boldsymbol{\beta}$-GP-EDC hydrogel: The CS-HLC/ $\beta$-GP-EDC hydrogel was prepared by mixing $5 \%$ 
(w/v) CS dissolved in $0.1 \mathrm{~mol} / \mathrm{L}$ acetic acid solution with an isocratic volume of $3 \%$ HLC solution. The mixture was stirred for $20 \mathrm{~min}$ in an ice-water bath until complete dissolution was achieved. Then, $1 \mathrm{~mL} 40 \% \beta$-sodium glycerophosphate $(\beta$ GP) and $0.02 \mathrm{~g}$ EDC was added drop wise until the $\mathrm{pH}$ of the solution reached 7.4. The solutions (sol) were placed in a thermo cell maintained 8 min for gelling at $37 \pm 0.5^{\circ} \mathrm{C}$.

Porous morphology of hydrogels: The surface and crosssectional morphologies of the hydrogels were studied by scanning electron microscopy using a JSM-5900LV (Japan).

Measuring gelling times: The gelling time was determined by the tube inverting method ${ }^{16,17}$ using a $10 \mathrm{~mL}$ centrifugal tube. About 3-5 mL of sol were placed in the centrifugal tube and inserted in a thermo cell until the solutions stopped flowing. The time that lapsed during this process was recorded as the gelling time.

Swelling measurement: The classical gravimetric method was employed to measure the swelling ratio of the hydrogels. For temperature-dependent swelling studies, gels were placed in triplicate in ultra-pure water and phosphate buffered saline solutions with $\mathrm{pH} 7.4$ at various temperatures for at least $24 \mathrm{~h}$ to reach equilibrium, after which the gels were weighed. The equilibrium swelling ratio was given as $\left(\mathrm{W}_{\mathrm{s}}-\mathrm{W}_{\mathrm{d}}\right) / \mathrm{W}_{\mathrm{d}}$, where $\mathrm{W}_{\mathrm{s}}$ and $\mathrm{W}_{\mathrm{d}}$ represent the weight of swollen gel and the dried gel weight, respectively.

in vitro Degradation: Degradation of hydrogels was performed in vitro using collagenase I with an activity of 0.5 $\mathrm{mg} / \mathrm{mL}$, hyaluronidase $(100 \mathrm{U} / \mathrm{mL})$ and trypsin $(100 \mathrm{U} / \mathrm{mL})$. The methods was followed from reference ${ }^{18}$.

Hematoxylin and eosin staining: After 1, 2 or 6 weeks, the mice were sacrificed and the tissue surrounding the hydrogels was removed and immediately fixed in $10 \%$ neutral buffered formalin. Using hematoxylin and eosin $(\mathrm{H}$ and E) staining to determined the property of hydrogels. The methods was come from Li et al. ${ }^{18}$.

\section{RESULTS AND DISCUSSION}

Hydrogel formation mechanism: EDC reaction with proteins have been reported by Tomihata and Ikada ${ }^{19}$ and Lu et $a .^{20}$, EDC containing $\mathrm{N}=\mathrm{C}=\mathrm{N}$ functional group, was used to react with the carboxyl groups on proteins or polysaccharides

A

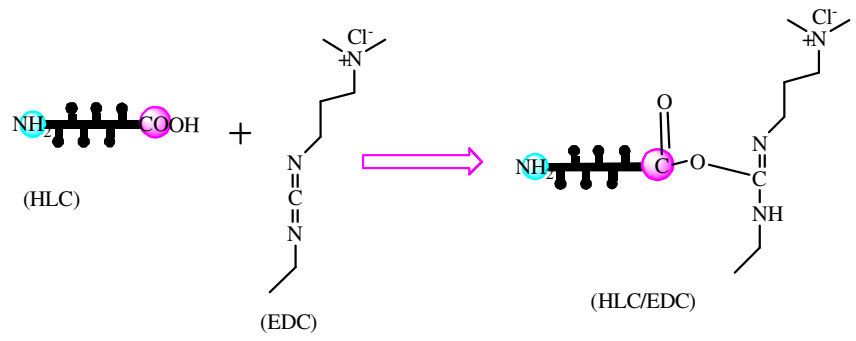

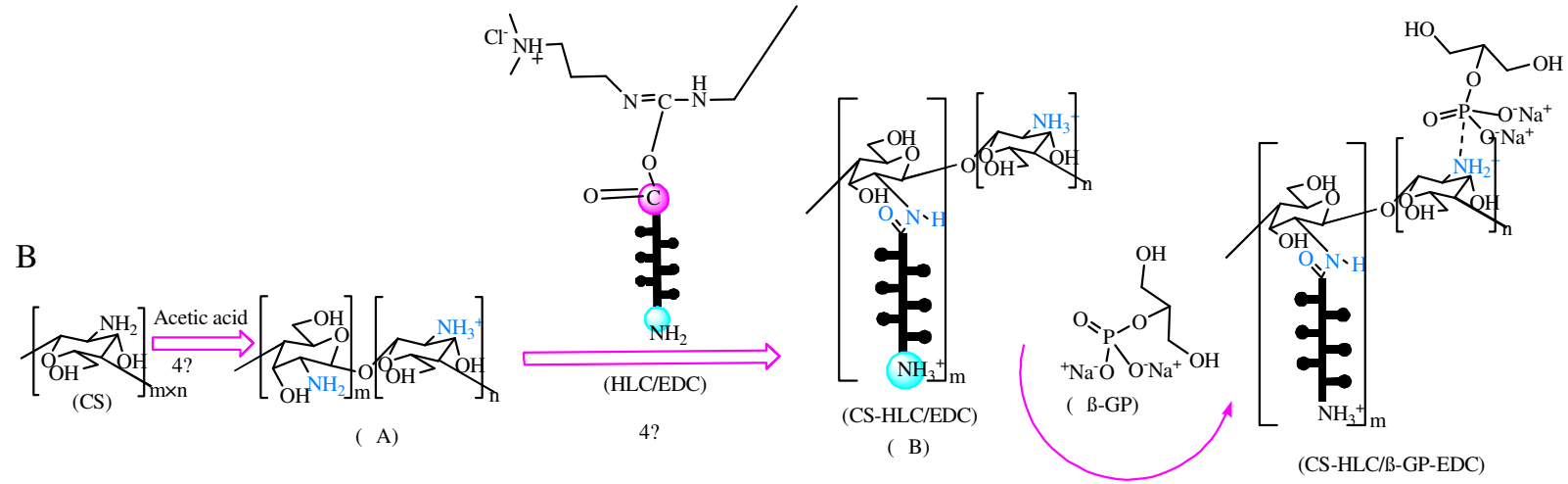

(C)

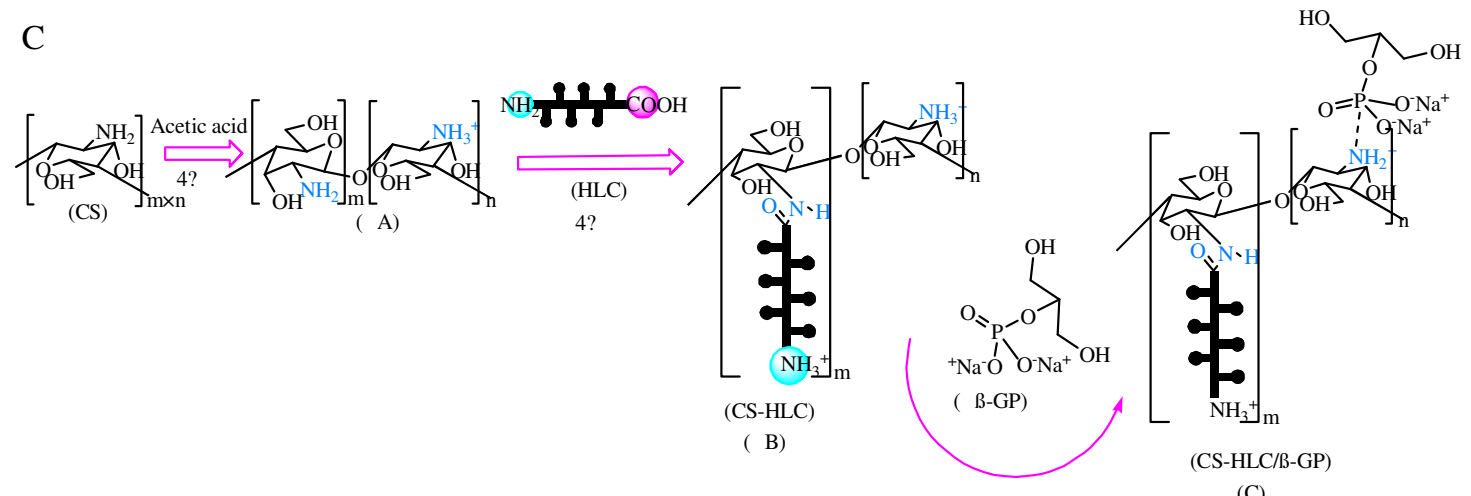

(C)

Scheme-I: Proposed interaction mechanisms for CS, HLC, EDC and $\beta$-GP: A, interaction of HLC and EDC; B, the CS-HLC/ $\beta$-GP-EDC hydrogel formation process; $\mathrm{C}$, the CS-HLC/ $\beta$-GP hydrogel formation process 
to form the unstable intermediate O-acylisourea. This intermediate makes proteins and polysaccharides reactive with other side groups to form an amide linkage between the amine and the acid (Scheme-IA). $-\mathrm{NH}_{2}$ of a part of CS chain was protonated to $-\mathrm{NH}_{3}{ }^{+}$under acidic conditions and also $-\mathrm{NH}_{2}$ of the end HLC chain was protonated to $-\mathrm{NH}_{3}{ }^{+}$. While part of $-\mathrm{NH}_{2}$ of not protonated CS reacted with HLC/EDC to glycoprotein chain was connected by a new amide bond (Scheme-IB). A new bound of $-\mathrm{NRH}_{2}{ }^{+}$were appeared mainly due to conjugation of $\mathrm{P}$ atoms on $-\mathrm{OPO}_{3}{ }^{2-}$ with $\mathrm{N}$ atoms from $-\mathrm{NH}_{3}{ }^{+}$of a part of CS chain (Scheme-IB). while the Scheme-IC have been reported by Li et al. ${ }^{21}$ compared with CS-HLC/ $\beta$-GP hydrogels, CS-HLC/ $\beta$-GP-EDC hydrogels was reaction by crosslinking agent of EDC, which was a water-soluble with good biocompatibility and easy removability from reaction systems, with may have the potential to produce a hydrogel with novel properties, including improved gelling time, swelling ratio, porous structure and good biodegradability.

Structure of hydrogels: The highly porous structure of these hydrogels was studied by SEM. CS-HLC/ $\beta-G P-E D C$ hydrogel showed complete formation of a jelly-like hydrogel whose appearance could be distorted under pressure (Fig. 1A). An irregular macroporous structure in the outer surface and non cross-connection in each pore-wall, the pore-walls in the structure of CS-HLC/ $\beta$-GP-EDC hydrogels were smoother and their surface structures were compact with uniform pore distribution, which renders them favorable for water and small molecule movement within the network (Fig. 1B).
A

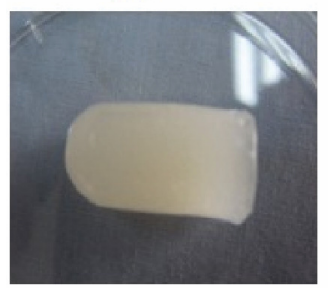

B

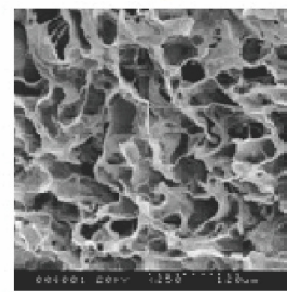

Fig. 1. Surface structure (A) and SEM (B) picture of CS-HLC/ $\beta-G P-E D C$ hydrogel

Gelling time: In order to store the products at reasonable time and shape with various temperature before injected into body, the relationship between the gelling time and temperature $\left(20-50{ }^{\circ} \mathrm{C}\right)$ was studied. At temperatures higher than $20^{\circ} \mathrm{C}$, gelling times decreased gradually with increasing temperature (Fig. 2). It including that CS-HLC/ $\beta$-GP-EDC hydrogel can be forming in situ hydrogel and dependence on temperature. Therefore, The CS-HLC/ $\beta$-GP-EDC hydrogel would be an ideal products used for plastic and reconstructive surgery of soft tissue.

Swelling measurement: In order to investigate of the influences of different media on the swelling of CS-HLC/ $\beta$ GP-EDC hydrogel, we studied the swelling ratios as a function of swelling media in double distilled water and phosphorus buffered saline $(\mathrm{pH}=7.4)$ as shown in Fig. 3. The swelling ratio of hydrogels in phosphorus buffered saline $(\mathrm{pH}=7.4)$ buffer solution was higher than it in double distilled water at same temperature with two fold. The CS-HLC/ $\beta$-GP-EDC hydrogel could realize sol-gel transition in suit and quickly

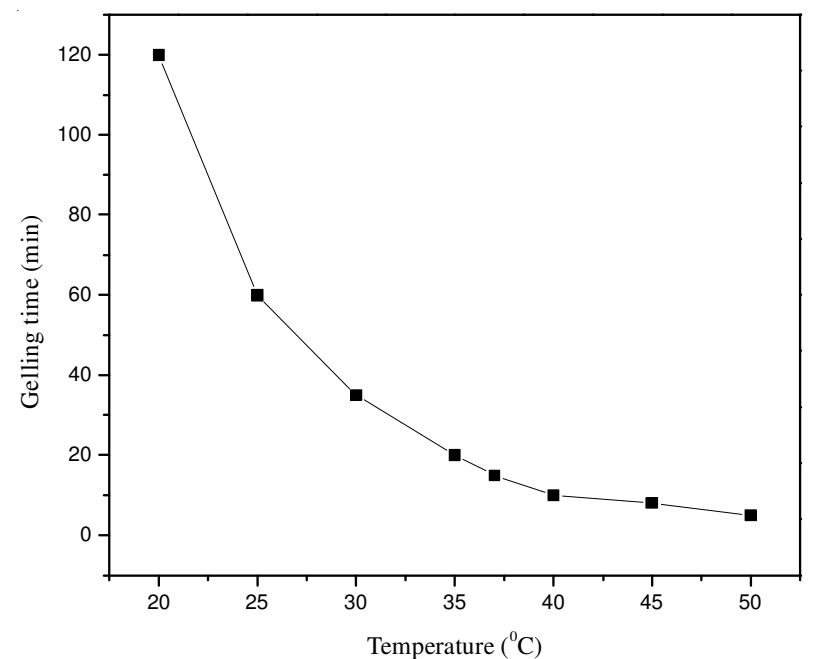

Fig. 2. Effects of temperature on the gelling time during the gelatin process

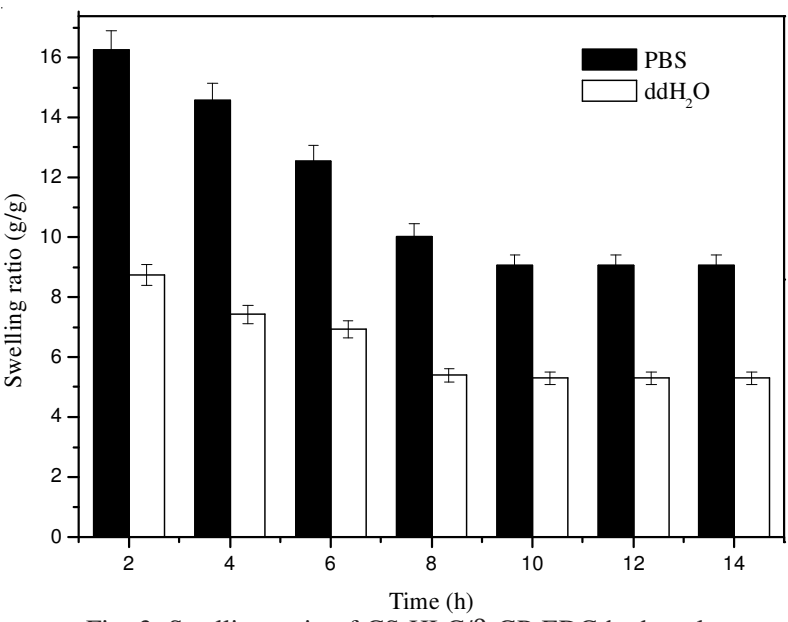

Fig. 3. Swelling ratio of CS-HLC/ $\beta$-GP-EDC hydrogel

adapt the temperature and $\mathrm{pH}$ of body fluid after injected into subcutaneous tissue. Therefore, the CS-HLC/ $\beta$-GP-EDC hydrogel had potential application in plastic and reconstructive surgery of soft tissue.

Enzymatic degradation in vitro: The resistance to degradation of the hydrogels was studied in vitro by examining weight loss with time in $10 \%$ (w/v) collagenase I hyaluronidase and trypsin. With long time, the weight loss was increased. weight loss increased $20 \%$ during the first 3 weeks and then increased $15 \%$ during the following weeks in $10 \%(\mathrm{w} / \mathrm{v})$ collagenase I, hyaluronidase and trypsin, approximately (Fig. 4). Therefore, the lowest rate of in vitro degradation was found in trypsin.

Hematoxylin and eosin staining: The histological response to injection of the hydrogels at 1,2 and 6 weeks was analyzed by hematoxylin and eosin staining (Fig. 5). It is clear from this image that hydrogels where gradually degraded in vivo. After 1 week, injection of CS-HLC/ $\beta$-GP-EDC hydrogel, the surrounding subcutaneous tissue were degenerated and necrotic (Fig. 5A). After 2 weeks, injection, the degree of inflammation was much milder and neutrophil infiltration was significantly decreased (Fig. 5B). After 6 weeks, inflammation was markedly reduced (Fig. 5C). It is indicating that the CS-HLC/ $\beta$-GP-EDC hydrogel was gradually adapting to the tissue surrounding the injection site. 


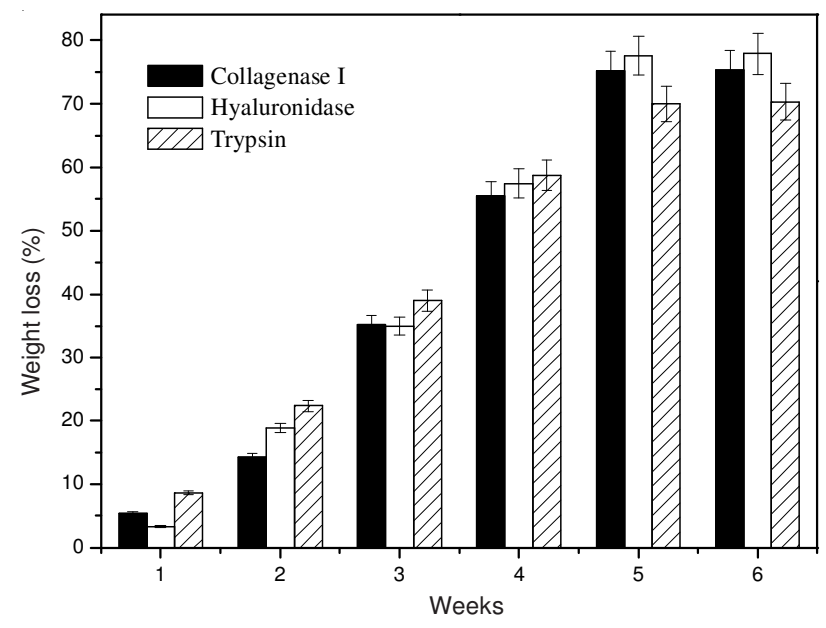

Fig. 4. In vitro enzymatic degradation of CS-HLC/ $\beta$-GP-EDC hydrogel

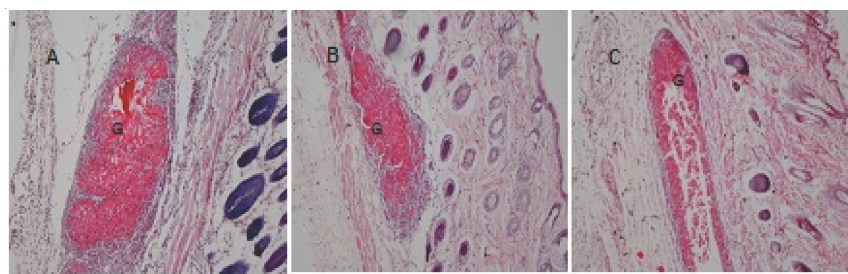

Fig. 5. Photomicrographs of the $\mathrm{H}$ and $\mathrm{E}$ staining, (A) 1 week after injection; (B) 2 weeks after injection; (C) 6 weeks after injection. $\mathrm{G}$ represent hydrogels

\section{Conclusion}

In this study, CS-HLC/ $\beta$-GP-EDC hydrogels were developed. This study demonstrated that the sol-gel transition temperature of the CS-HLC/ $\beta$-GP-EDC hydrogel was within the range of $36-37^{\circ} \mathrm{C}$ at neutral $\mathrm{pH}$ value and with crosslinking agent of EDC, the gelling time was shortened. Furthermore, the swelling equilibrium of CS-HLC/ $\beta$-GP-EDC hydrogels was reached at approximately after $10 \mathrm{~h}$, which could easily be adapted to body fluid. Additionally, the CS-HLC/ $\beta-G P-$ EDC hydrogels had a highly porous structure which would provide more space for water and small molecules to move freely in the network. Results also showed that the morphology of the injected gel resisted change for a relatively long time in vivo and degraded slowly over time. In summary, the CS-HLC/ $\beta$-GP-EDC hydrogel was proven to be highly suitable as an injectable material, as well as a tissue engineering scaffold.

\section{ACKNOWLEDGEMENTS}

This study was financially supported by the National Natural Science Foundation of China (21176200, 21276210, 21106112, 21206135, 31000019 and 21106111), China Postdoctoral Science Foundation (20110490171), China Postdoctoral Science Special Foundation (2012T50755), the Scientific Research Program of Shaanxi Provincial Department of Education, China (2013JK0696, 12JK0449, 12JS099, 12JS100, 12JS101, 2012JC23 and 11JS102); Shaanxi Provincial Scientific Technology Research and Development Program (2012KJXX-28, 2013KJXX-28, 2012JM2014, 2011JE003, 2011JQ4026 and 2010JQ2012) and the NWU Graduate Innovation and Creativity Funds (YZZ12037) and the Shaanxi Key Subject Program, China.

\section{REFERENCES}

1. J.S. Temenoff and A.G. Mikos, Biomaterials, 21, 2405 (2000).

2. J.L. Drury and D.J. Mooney, Biomaterials, 24, 4337 (2003).

3. Q. Hou, P.A. De Bank and K.M. Shakesheff, J. Mater. Chem., 14, 1915 (2004).

4. A.B. Pratt, F.E. Weber, H.G. Schmoekel, R. Muller and J.A. Hubbell, Biotechnol. Bioeng., 86, 27 (2004).

5. Q. Hou, P.A. De Bank and K.M. Shakesheff, J. Mater. Chem., 14, 1915 (2004).

6. A. Di Martino, M. Sittinger and M.V. Risbud, Biomaterials, 26, 5983 (2005).

7. S.E. Kim, J.H. Park, Y.W. Cho, H. Chung, S.Y. Jeong, E.B. Lee and I.C. Kwon, J. Control. Rel., 91, 365 (2003).

8. H. Tan, Y. Gong, L. Lao, Z. Mao and C. Gao, J. Mater. Sci. Mater. Med., 18, 1961 (2007).

9. D.D. Fan, Y. Mi and J.R. Song, Chin. J. Chem. Eng., 10, 752 (2002).

10. X.F. Hua, D.D. Fan, Y.E. Luo, X. Zhang, H.J. Shi, Y. Mi, X. Ma, L. Shang and G. Zhao, Chin. J. Chem. Eng., 14, 242 (2006).

11. Y. Zhai, F.Z. Cui and Y. Wang, Curr. Appl. Phys., 5, 429 (2005).

12. Y. Wang, F.Z. Cui, Y. Zhai, X.M. Wang, X.D. Kong and D.D. Fan, Mater. Sci. Eng. C, 26, 635 (2006).

13. Y.Y. Yu and D.D. Fan, Asian J. Chem., 23, 1548 (2011).

14. P.M. Santos, J.G. Winterowd, G.G. Allen, M.A. Bothwell and E.W. Rubel, Head Neck Surg., 105, 12 (1991).

15. C.H. Zhu, D.D. Fan, Z.G. Duan, W.J. Xue and L.A. Shang. J. Biomed. Mater. Res. Part A, 89, 829 (2009).

16. H.J. Chung, D.H. Go, J.W. Bae, I.K. Jung, J.W. Lee and K.D. Park, Curr. Appl. Phys., 5, 485 (2005).

17. F. Ganji, M.J. Abdekhodaie and A. Ramazani S.A., J. Sol-Gel Sci. Technol., 42, 47 (2007).

18. X. Li, X.X. Ma, D.D. Fan and C.H. Zhu, Soft Matter, 8, 3781 (2012).

19. K. Tomihata and Y. Ikada, J. Biomed. Mater. Res., 37, 243 (1997).

20. G.Y. Lu, L.J. Kong, B.Y. Sheng, G. Wang, Y. Gong and X. Zhang, Eur. Polym. J., 43, 3807 (2007).

21. X. Li, D.D. Fan, X.X. Ma, C.H. Zhu, Y.N. Luo, B.W. Liu and L. Chen, Soft Mater, 12, 1 (2014). 